\title{
Dosimetric Performance of A-Si Electronic Portal Imaging Devices
}

\author{
Mukhtar Alshanqity ${ }^{*}$, Andrew Nisbet ${ }^{2}$ \\ ${ }^{1}$ Medical Physics Department, Comprehensive Cancer Centre, King Fahad Medical City, Riyadh, Saudi Arabia \\ ${ }^{2}$ Centre for Nuclear and Radiation Physics, Physics Department, University of Surrey, Guildford, UK \\ Email: "malshanqity@kfmc.med.sa
}

Received 26 March 2016; accepted 28 May 2016; published 31 May 2016

Copyright (C) 2016 by authors and Scientific Research Publishing Inc.

This work is licensed under the Creative Commons Attribution International License (CC BY).

http://creativecommons.org/licenses/by/4.0/

(c) (i) Open Access

\section{Abstract}

The majority of EPID dosimetry literature discusses response linearity and the so-called image lag and ghosting effects despite the lack of a common definition of these quantities. However, the results of these studies are generally not consistent, and it is often difficult to compare the results from different studies. We present here a detailed study of the acquisition and readout characteristics of a-Si EPID and its dosimetric performance. EPID response was assessed over the range of 1 - $500 \mathrm{MU}$ using different dose rates and integration times. In addition, a computer model was designed to simulate the EPID image formation with different dose, dose rate, and integration time combinations. All aspects of image processing and readout simulation were carried out using custom written MatLab codes. Two distinct signal profiles were observed depending on the delivered dose, dose rate and integration time combination. Total integrated signal $\left(\mathrm{S}_{\mathrm{T}}\right)$ is linear with the delivered dose. For dosimetry, image lag and ghosting effects mainly result in the residual signal $\left(S_{R}\right)$ that appears as delayed signal after the end of irradiation. At its maximum, $S_{\mathrm{R}}$ is less than $2.5 \%$ of $S_{\mathrm{T}}$. The readout technique is such that it is impossible to measure $S_{R}$ accurately. $S_{R}$ is definable only when readout equilibrium occurs. Signal profiles provide a through and reliable description of the EPID response incorporating the dose, dose rate, integration time, and the residual signal. The definition of EPID signals based on this method shall provide an accurate universal EPID dosimetry framework.

\section{Keywords}

EPID, Portal Dosimetry, Radiotherapy QA

\section{Introduction}

Electronic portal imaging devices (EPID) were introduced initially for patient imaging purposes; these included

${ }^{*}$ Corresponding author.

How to cite this paper: Alshanqity, M. and Nisbet, A. (2016) Dosimetric Performance of A-Si Electronic Portal Imaging Devices. International Journal of Medical Physics, Clinical Engineering and Radiation Oncology, 5, 162-175. 
patient immobilization and treatment field verifications [1]. The application of EPID for dosimetry has been investigated from the first camera based EPID through scanning liquid filled ion chamber (SLIC) EPID generation to the most recent, and commonly used, amorphous silicon (a-Si) based EPID [1] [2]. Nowadays, EPID are widely used for routine quality assurance and dosimetry verifications in addition to their main imaging role. The number of published articles concerned with EPID dosimetry has risen significantly in the last decade after the introduction of amorphous silicon based EPID with a noticeable trend towards in vivo and three dimensional dosimetry applications [1]. The reported main advantages of a-Si EPID over the older generations are the linearity, stability, higher resolution and flexibility. On the other hand, they suffer from so-called image lag and ghosting effects [1]-[6]. Numerous articles have been published regarding the calibration, performance and different approaches for EPID dosimetry implementation. These have mainly focused on EPID linearity with radiation dose and the effects of image lag and ghosting, and have rarely referred to the effect of EPID integration time and readout technique [2]-[24]. There is no single definition or criterion for the measurement of the EPID signal, image lag, or ghosting. Moreover, the reported dosimetric characteristics of a-Si EPID in the literature vary considerably. A recent publication [24], the first and only one found that clearly acknowledges the effect of readout technique, has suggested that these effects are related to the small radiation doses and has concluded that EPID response should be corrected with such radiation doses. Some of the other published research methods describe and quantify the EPID response in terms of the EPID measured signal from fixed delivered radiation dose with variable dose rates and acquisition parameters, and fixed irradiation time and variable radiation doses and acquisition parameters [2] [24]. This is an over simplification that leads to inaccurate characterization of the EPID signal where the readout technique and integration time effects are overlooked and confused with dose and dose rate effects. Other researchers have analyzed the EPID signal as a function of dose per frame [2] [8] and concluded that EPID response is nonlinear as a function of dose and dose rate due to image lag and ghosting [2]. In another article, the authors have noticed inconsistent EPID response with small MU settings and correlated it to the ghosting effect [8]. Winkler et al. [19] have defined a radiation dose of $30 \mathrm{MU}$ as a threshold for small doses and reported different EPID response between small and large radiation doses and suggested a logarithmic function to describe EPID response in terms of dose and dose rate. The authors, in another paper [18], have indicated that the EPID response is "by far not ideal" due to two reasons; first, the non-linear relationship between frame signal and the dose per frame and the second is the linac start-up effect. Vial et al. [11] concludes that corrections are required for the non-linear EPID response in the small MU range. They, however, have not defined a value for small MU range, but the figure they presented shows non-linear EPID signal per MU beyond $100 \mathrm{MU}$. McCurdy et al. [6] showed that the EPID signal per MU is dose dependent for both integrated and continuous acquisition modes with integrated signal mode being the closest to linear response. In addition, they have suggested a correction method to compensate for the missing signal in order to achieve linearity especially in the low dose range.

Nevertheless, many recent publications [9] [13] discuss the use of EPID as in vivo dosimeters giving an impression of a well-established EPID characterization and calibration. In many of these publications, the manufacturer definition of the integrated image has been used to represent the EPID signal. A thorough discussion of the different approaches used for EPID dosimetry may be found in the literature review carried out by van Elmpt et al. [1].

EPID devices are calibrated by correlating measured EPID response to the radiation dose either in terms of linac monitor units or measured absorbed dose in Gray (Gy). Linear accelerator calibration is well regulated and there are different dosimetry codes of practice in different parts of the world that differ in theory and technique but aim to correlate linac output to traceable and comparable primary standards. However, the EPID response has a less well-defined general description as the signal from the start to the end of the irradiation and there is no consensus view on the effect of the so-called image lag and ghosting effects.

In this study, we characterize the EPID response in terms of the signal profile incorporating the influence of time, dose, dose rate, readout technique and detector gain setting. We demonstrate the extent of the influence of the detector readout technique on the EPID signal and its interference with detector gain, image lag and ghosting effects. Based on these results, a novel framework for EPID dosimetry is recommended.

\section{Methods and Materials}

\subsection{The iViewGT EPID}

Two iViewGT EPID with Perkin Elmer Type XRD 1640 flat panel amorphous silicon detectors have been em- 
ployed. The Perkin Elmer XRD unit is a standalone radiation detector containing the a-Si flat panel detector and its electronics in aluminum housing. Each EPID is attached to an Elekta Synergy Linac. The iViewGT is mounted at $160 \mathrm{~cm}$ from the linac's target when in operation, with a detector area of $41 \times 41 \mathrm{~cm}^{2}$ and $1024 \times$ 1024 pixels. This results in a useful field size of $25 \times 25 \mathrm{~cm}^{2}$ at the isocentre plane. The two linacs were calibrated and maintained to produce $1.00 \pm 0.03 \mathrm{cGy}$ at the reference depth for each machine monitor unit (MU) for $10 \times 10 \mathrm{~cm}^{2}$ field at $100 \mathrm{~cm}$ focus to skin distance (FSD). Both linacs are calibrated using ionization chambers with calibration factors traceable to a primary standard.

The flat panel detector measuring element is an amorphous Silicon thin film array on a glass substrate, in which each pixel is a photodiode and a thin film transistor (TFT) switch in one circuit. Visible light photons resulting from the conversion of $\mathrm{x}$-rays in the scintillator create electron-hole pairs in the photodiode array. These electrons are collected by a bias voltage and stored in the photodiode capacitors ready for readout when the TFT switch is closed. The TFT switches are controlled by the row driver which is programmed to release the accumulated charge in the individual pixel capacitors of a whole row of pixels at a time when triggered by turning the TFT switches on and off. The pixel array is divided into separate readout groups; each group is connected to the readout electronics via a multiple channel line. For the XRD 1640, there are 16 readout groups and 128 channels for each group, making a total of 1024 columns of pixels where each column also consists of 512 pixels. The 16 readout groups are clustered in two electrically separated parts. The upper part controls readout groups 1 through 8, and the lower part controls readout groups 9 through 16, illustrated in Figure 1. The row driver scans TFT switches from left to right in the upper readout group and from right to left in the lower group. In each scan the accumulated charge in a whole row of pixels is released and transferred to the readout circuit. Two rows of pixels are readout simultaneously from both upper and lower readout groups of the detector each time the row driver is invoked, then amplified and stored in the readout electronics' capacitors of the frame grabber. Each pixel in the array is a separate entity that has its own electrical properties and radiation sensitivity. In addition, different readout groups are controlled by different subsystems in the detector panel.

Bad pixels that are dead or give inconsistent readings are corrected using an updated manufacturer provided bad pixels map unique to each device. In addition, each and every pixel has an individual intrinsic measurable signal resulting in an elevated measured signal value, this is referred to as dark or offset current. In this study, at least five frames were allowed before starting the radiation beam to be used for offset correction, and the acquisition is continued after the end of irradiation to the maximum possible number of frames. Variable pixel gain across the imager is normalized in gain calibration, which is performed by averaging a sequence of bad pixels

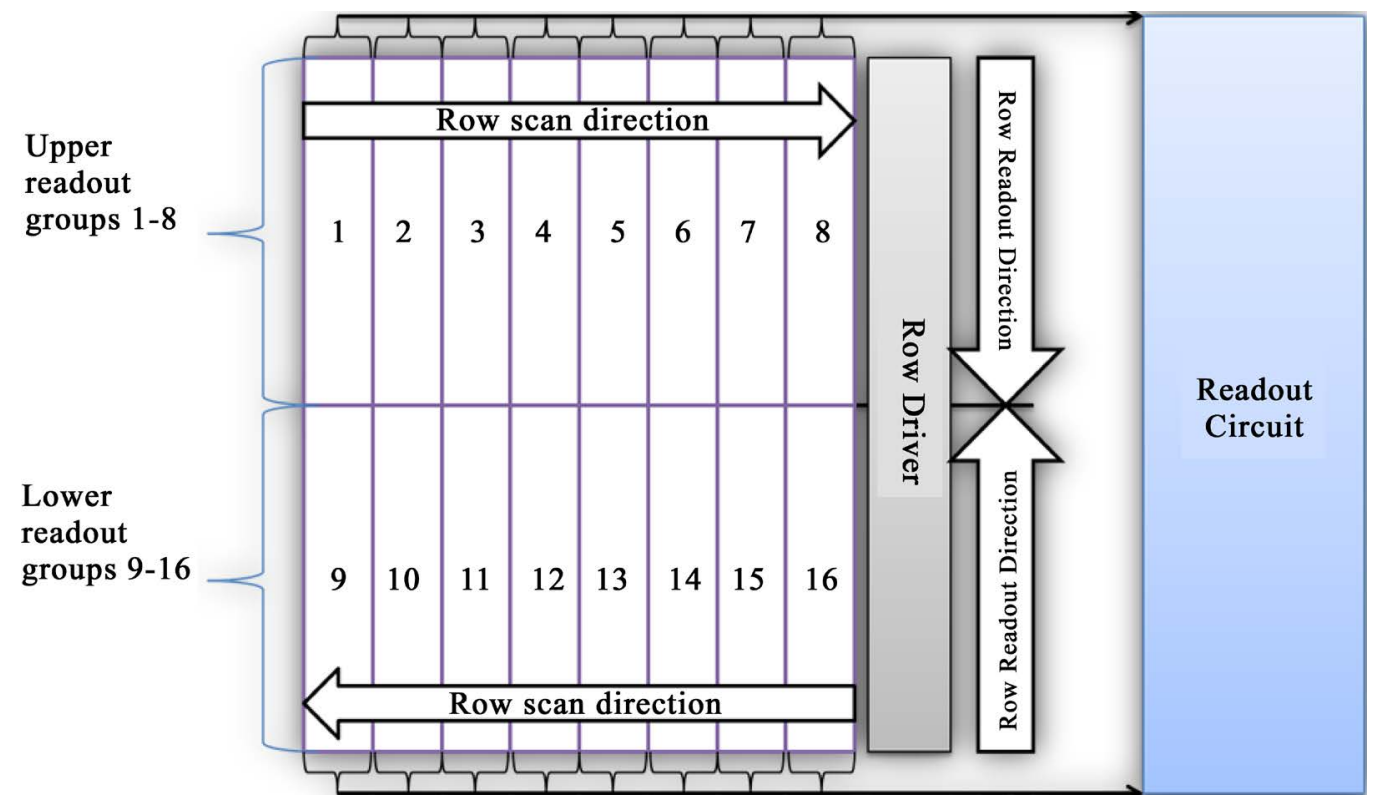

Figure 1. Schematic of the XRD 1640 readout, the detector is divided in two separate upper and lower parts. Each part contains 8 independent readout groups. Readout takes place row by row across the readout groups starting from the upper and lower ends of the detector. The upper readout groups are scanned from left to right and the lower ones from right to left. 
and offset corrected images that have been irradiated with an open, uniform beam for the whole area of the imager. The median of all the pixels is calculated and taken as a reference to create a gain correction image. However, the gain calibration removes the beam profile information resulting in an optimally flat beam profile. To correct for this the measured two-dimensional beam profile at depth of interest (i.e. $d_{\max }$ ) is normalized to the central axis (CAX) dose and applied to the EPID measurements to maintain dosimetric accuracy. Each EPID measurement is corrected for the Linac output fluctuation using the measured Linac output on the day of measurement.

\subsection{EPID Data Acquisition}

The EPID response was assessed over the clinical range of interest from 1 to $500 \mathrm{MU}$. EPID measured data were captured by the dedicated iViewGT computer using its X-ray imaging software (XIS), without any further corrections, as 16 bit depth raw Heinemann imaging software (HIS) files. The maximum acquisition time is limited by hardware capabilities, which in both EPID devices were enough to accommodate approximately 200 frames. All measurements were carried out using the maximum number of frames to study the EPID signal behavior after the end of irradiation. EPID signal was taken as the average of the $25 \times 25$ pixels around the central axis. Signal quality index $\left(\mathrm{S}_{\mathrm{QI}}\right)$ was evaluated for all measurements [25]. All corrections, Linac output, bad pixels, offset and gain corrections, and further processing and analysis were carried out using custom written MatLab codes.

\subsection{EPID Computer Model}

During image acquisition with the iViewGT EPID, peculiar patterns may be observed at the beginning of radiation beam delivery and at the end of irradiation. As the radiation beam starts, the first few frames show a gradient in the radiation beam intensity across the field with the highest intensity at the center of the detector and the lowest towards the two ends of the detector along the Y-axis as shown in Figure 2. This figure demonstrates a greyscale image analogous to that observed in the XIS software on the iViewGT terminal during actual measurements. After the first few frames, iViewGT images exhibit a uniform radiation field. At the end of irradiation, a similar but converse pattern is observed with the highest intensities now at the two ends and the lowest intensity at the center of the EPID as shown in Figure 2. These patterns are observed in all measurements, regardless of the EPID employed, dose, dose rate, integration time, field size and whether an imaging object is present or not. Furthermore, the gradient always starts at the center of the EPID even with off centered and asymmetric fields. To investigate, and visualize, the effect of the iViewGT image formation process on the measurements, a computer model for the EPID was designed using MatLab.

The EPID computer model simulates the radiation pulses at the same Linac pulse repetition frequency, and the readout of the EPID at the same frequency as the iViewGT. The user defines the radiation dose to be delivered in terms of MU and the field size as a square field directly in the code or as a complex field with the aid of another routine. Dose rate and integration time are selectable from a predefined list for the Linac in use.
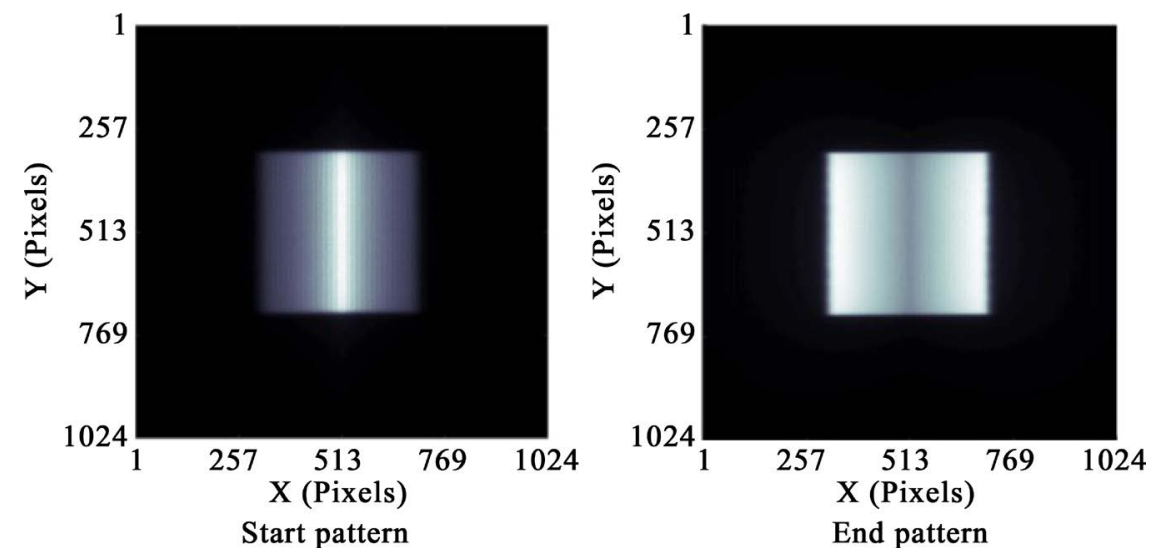

Figure 2. The start-up (left) and end (right) patterns observed on the iViewGT computer. The first few frames always show a similar pattern regardless of dose, dose rate, integration time and radiation field geometry. The end pattern is similar to the start-up pattern but with reversed gradient. 


\section{Results}

\subsection{EPID Signal Profile}

Once the EPID is irradiated, the pixel readouts increase dramatically, depending on dose rate and integration time, to a higher value on the dynamic range (approximately 9000 for EPID-1 and 40,000 for EPID-2, both used with the fastest integration time and highest dose rate). The readout then continues to reach a maximum by the end of irradiation. After which the signal decays rapidly, this can be seen as a decaying tail in Figure 3 . The total accumulated signal value of an irradiated pixel depends on the delivered dose only, and the signal value in a particular frame depends on the dose delivered during the integration time, or put simply is a function of both dose rate and integration time. The corrected EPID image signal profile Figure 3, shows the zeroed baseline before radiation start, the EPID response signal during irradiation and the slow release of the residual signal $\left(\mathrm{S}_{\mathrm{R}}\right)$ after the end of irradiation. Distinguishable features of the EPID signal profile are labeled to simplify the description of the EPID response, the label names and descriptions are summarized in Table 1. Each signal profile

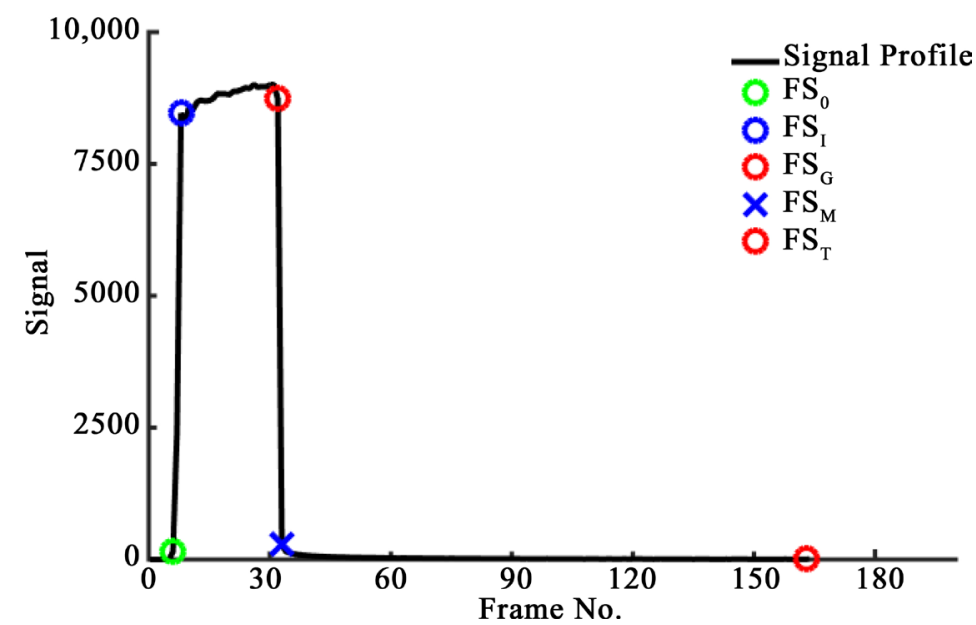

Figure 3. Signal profile from EPID-1 after bad pixel, offset and gain corrections for $100 \mathrm{MU}$ at a dose rate of $540 \mathrm{MU} / \mathrm{min}$ and using 0.433 second integration time. The frame first signal after irradiation $\mathrm{FS}_{0}$, the frame initial maximum signal $\mathrm{FS}_{\mathrm{I}}$, frame global maximum $\mathrm{FS}_{\mathrm{G}}$, frame maximum $\mathrm{FS}_{\mathrm{M}}$ and terminal maximum $\mathrm{FS}_{\mathrm{T}}$ signals are highlighted.

Table 1. List of abbreviations and definitions of signals used to describe the signal profile.

\begin{tabular}{|c|c|}
\hline Name & Discretion \\
\hline $\mathrm{FN}_{0}$ & Frame number of the first measurable signal \\
\hline $\mathrm{FN}_{\mathrm{I}}$ & Frame number of the initial maximum signal \\
\hline $\mathrm{FN}_{\mathrm{G}}$ & Frame number of the global maximum signal \\
\hline $\mathrm{FN}_{\mathrm{M}}$ & Frame number of the final maximum signal \\
\hline $\mathrm{FN}_{\mathrm{T}}$ & Frame number of the last measurable signal \\
\hline $\mathrm{FS}_{0}$ & The signal from the first measurable frame \\
\hline $\mathrm{FS}_{\mathrm{I}}$ & The frame signal of the initial maximum \\
\hline $\mathrm{FS}_{\mathrm{G}}$ & The frame signal of the global maximum \\
\hline $\mathrm{FS}_{\mathrm{M}}$ & The frame signal of the final maximum \\
\hline $\mathrm{FS}_{\mathrm{T}}$ & The frame signal of the total maximum \\
\hline $\mathrm{FS}_{\mathrm{P}}$ & Peak signal \\
\hline $\mathrm{S}_{\mathrm{T}}$ & Total Integrated Signal \\
\hline $\mathrm{S}_{\mathrm{I}}$ & Integrated Signal to the Initial maximum \\
\hline $\mathrm{S}_{\mathrm{M}}$ & Integrated Signal to the final maximum plus the first frame after $\mathrm{FS}_{\mathrm{G}}$ \\
\hline $\mathrm{S}_{\mathrm{R}}$ & Integrated signal from the second frame after $\mathrm{FS}_{\mathrm{G}}$ to $\mathrm{FS}_{\mathrm{T}}$ \\
\hline MES & Mean equilibrium signal \\
\hline $\mathrm{SQ}_{\mathrm{I}}$ & Signal quality index \\
\hline
\end{tabular}


feature $(\mathrm{X})$ is associated with three different values, a frame number $\left(\mathrm{FN}_{\mathrm{X}}\right)$, a frame signal $\left(\mathrm{FS}_{\mathrm{X}}\right)$, and an integrated signal $\left(\mathrm{S}_{\mathrm{X}}\right)$.

The observed EPID response is delayed until after the beginning of irradiation by at least one integration time. The first completed frame after starting the radiation $\left(\mathrm{FN}_{0}\right)$ contains the first measurable frame signal $\left(\mathrm{FS}_{0}\right)$, the value of this signal is variable and depends on the coincidence of frame readout with the start of irradiation. The shorter the time between the beginning of frame formation and the start of the radiation beam the higher the $\mathrm{FS}_{0}$ value. $\mathrm{FN}_{0}$ is fixed at a value of six in this study for presentation purpose only. After several frames, or integration times, the frame signal increases to a much higher value, the initial maximum frame signal $\left(\mathrm{SF}_{\mathrm{I}}\right)$, at the frame number $\mathrm{FN}_{\mathrm{I}}$. The value of $\mathrm{SF}_{\mathrm{I}}$ is found to be independent of the delivered radiation dose and constant for a given dose rate and integration time combination, Figure 4. The value of $\mathrm{SF}_{\mathrm{I}}$ is also dependant on the electronic gain setting of the EPID. EPID-1 was set for a low gain setting resulting in an $\mathrm{SF}_{\mathrm{I}}$ value of around $14 \%$ of the dynamic range and EPID-2 was set for a higher gain with an $\mathrm{SF}_{\mathrm{I}}$ value of around $60 \%$ of the dynamic range, both at the highest dose rate and fastest integration time. The signal profile of EPID-1 is similar for all the dose range and exhibits a steady and slow increase during irradiation shown in Figure 3. EPID-2 on the other hand has shown two different patterns during irradiation, one for dose range lower than $150 \mathrm{MU}$ in which the signal profile is almost constant during irradiation, and the other is a signal profile with slightly decreasing values during irradiation for the rest of the dose range.

The last complete frame formed during irradiation is the frame of the global maximum $\left(\mathrm{FN}_{\mathrm{G}}\right)$, the accumulated signal during this frame's integration time is the global maximum signal $\left(\mathrm{FS}_{\mathrm{G}}\right)$, and the total integrated EPID signal until the last frame prior to the end of irradiation is the global integrated signal $\left(\mathrm{S}_{\mathrm{G}}\right)$. The frame immediately following is the frame of maximum signal $\left(\mathrm{FN}_{\mathrm{M}}\right)$, with the maximum frame signal $\left(\mathrm{FS}_{\mathrm{M}}\right)$ and the maximum integrated signal $\left(\mathrm{S}_{\mathrm{M}}\right)$. However, radiation pulses may end at any point during the integration time of this frame. Hence, $\mathrm{FS}_{\mathrm{M}}$ signal will always be less than the preceding $\mathrm{FS}_{\mathrm{G}}$ frame signal. The integrated EPID signal after $\mathrm{FS}_{\mathrm{M}}$ to the last frame with a measurable signal above the baseline $\left(\mathrm{FN}_{\mathrm{T}}\right)$ represents the residual signal $\left(\mathrm{S}_{\mathrm{R}}\right)$.

The total integrated EPID signal $\left(\mathrm{S}_{\mathrm{T}}\right)$ is measured from the first frame $\mathrm{FN}_{0}$ to last measurable frame $\mathrm{FN}_{\mathrm{T}}$; it is also equal to the sum of $S_{M}$ and $S_{R}$. The shape of the signal profile is different for short irradiations and appears to have a Gaussian shape rather than the typical shapes described in Figure 5.

Short irradiations' signal profiles are observed when the radiation dose is delivered in a time period less than twice the integration time. All frame readouts then are composed of signals from irradiated pixels and baseline values because irradiation does not last long enough for a single frame to be formed. In such scenarios, the whole radiation dose is delivered in one or two frames while the EPID response is observed a few frames after the end of the radiation delivery. The main features of such signal profiles are the first frame signal $\left(\mathrm{FS}_{0}\right)$, peak frame signal $\left(\mathrm{FS}_{\mathrm{P}}\right)$ and the last frame signal $\left(\mathrm{FS}_{\mathrm{T}}\right)$. The previously described features are not distinguishable, especially the residual signal since the whole EPID response is observed to be measured after the end of irradiation. Peak signal increases uniformly with delivered radiation doses Figure 6, to reach a maximum value close

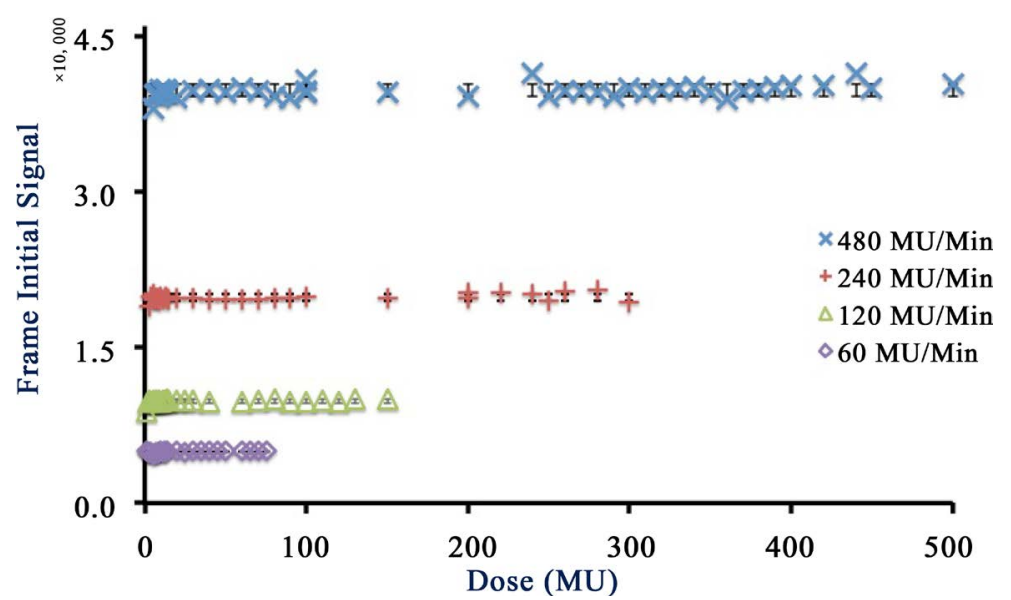

Figure 4. Initial frame signal $\left(\mathrm{FS}_{\mathrm{I}}\right)$ extracted from EPID-2 measurements as a function of the delivered radiation dose, $\mathrm{FS}_{\mathrm{I}}$ is constant for a given dose rate and integration time combination. 


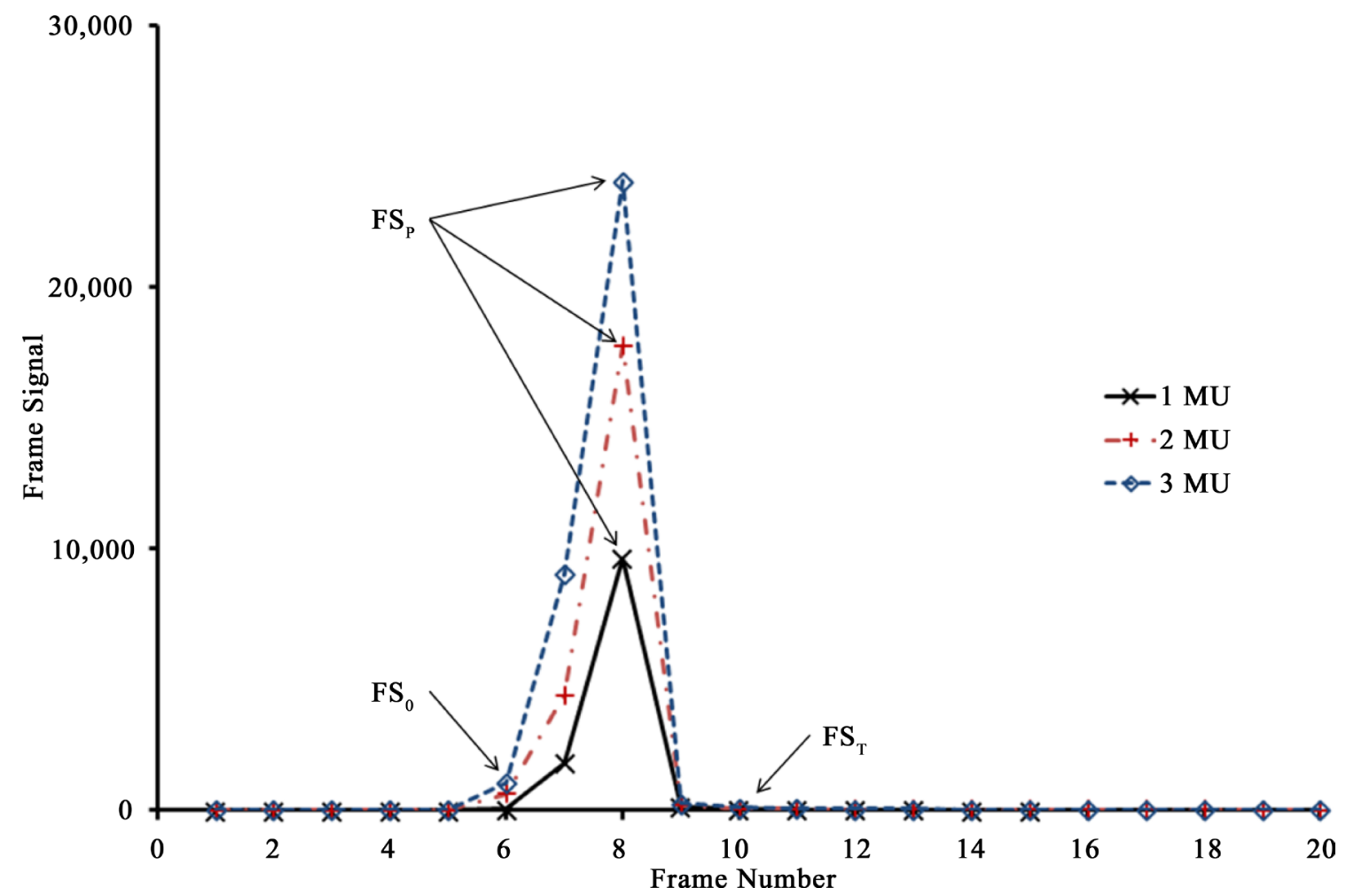

Figure 5. The signal profiles for 1, 2 and $3 \mathrm{MU}$ radiations with a dose rate of $540 \mathrm{MU} / \mathrm{min}$ and $0.433 \mathrm{~s}$ integration time. With such short irradiations the EPID response appears after the completion of radiation delivery which takes a fraction of the integration time, the signal profile is different from that with longer irradiations.

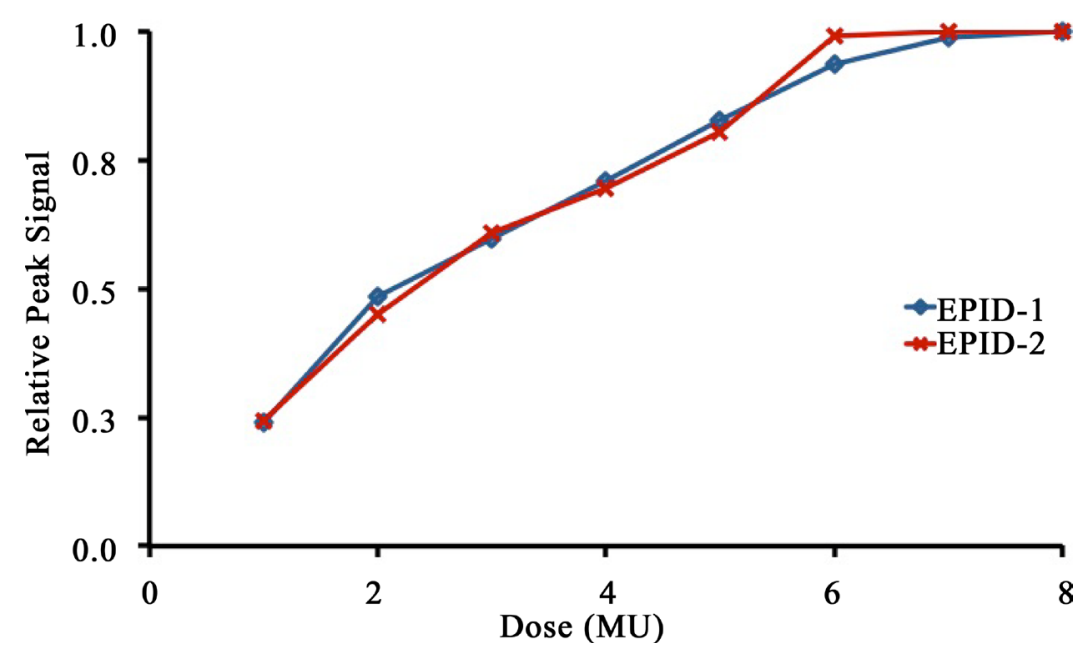

Figure 6. The value of the peak signal relative to the respective $\mathrm{FS}_{\mathrm{I}}$ with equilibrium index equal to one with the same dose rate and integration time for EPID-1 and EPID-2. FS $\mathrm{P}_{\mathrm{P}}$ increases with the delivered radiation dose to reach a maximum value close to the respective $\mathrm{FS}_{\mathrm{I}}$. Both curves are showing the same $\mathrm{FS}_{\mathrm{P}}$ behaviour except that EPID-1 values are much lower than EPID-2 due to the difference in the detector gain settings. Also the readout equilibrium is achieved at lower dose with EPID-2 due to the difference in the reference calibration point between the two Linacs, which results in lower Linac-2 dose rate compared to Linac-1. The dose range is from 1 to $8 \mathrm{MU}$, the dose rate is $540 \mathrm{MU} / \mathrm{min}$ for EPID-1 and $480 \mathrm{MU} / \mathrm{min}$ for EPID-2, and the integration time is $0.433 \mathrm{~s}$.

to that of $\mathrm{FS}_{\mathrm{I}}$ in longer irradiations. The increase in the peak signal value is more dramatic with smaller MU settings and less with higher MU settings. The highest peak signal value corresponds to a radiation beam delivered in a time equivalent to twice the integration time. That value is very close to $\mathrm{FS}_{\mathrm{I}}$ in longer irradiations. 


\subsection{EPID Dose Response}

The most important dosimetric parameter obtained from the EPID signal profile is the total integrated signal $\left(\mathrm{S}_{\mathrm{T}}\right)$ that represents the total measurable radiation induced charge in the EPID. It is important to emphasize that $\mathrm{S}_{\mathrm{T}}$ is the total 'measurable' EPID signal and not necessarily the total radiation induced signal. This is a result of hardware limitations, particularly for long irradiations where the EPID signal cannot be integrated to the point where frame readout reaches baseline value [25].

$\mathrm{S}_{\mathrm{T}}$ is linear with the delivered radiation dose across the whole measurement range, including the small doses range, independent of dose rate and integration time. Figure 7 shows $\mathrm{S}_{\mathrm{T}}$ as a function of the delivered radiation dose for the range from 1 to $600 \mathrm{MU}$, including measurements with all different dose rates and integration times available. The small doses range, from 1 to $10 \mathrm{MU}$, is expanded on the subset frame in the same figure. As a result of the EPID linear response to the delivered radiation dose, the EPID signal per MU is found to be constant and independent of delivered radiation dose, dose rate and integration time. For EPID-1 the mean value for $\mathrm{S}_{\mathrm{T}}$ per MU is $2380.0 \pm 0.2$, and for EPID-2 the mean is 11,618.0 \pm 1.2 . Both dose rate and integration time have no observable influence on $\mathrm{S}_{\mathrm{T}}$ beyond measurement uncertainties. However, dose rate and integration time do affect $\mathrm{FS}_{\mathrm{I}}$ values shown in Figure 4. The frame signal during irradiation is linearly proportional to both the dose rate and integration time, Figure 8.

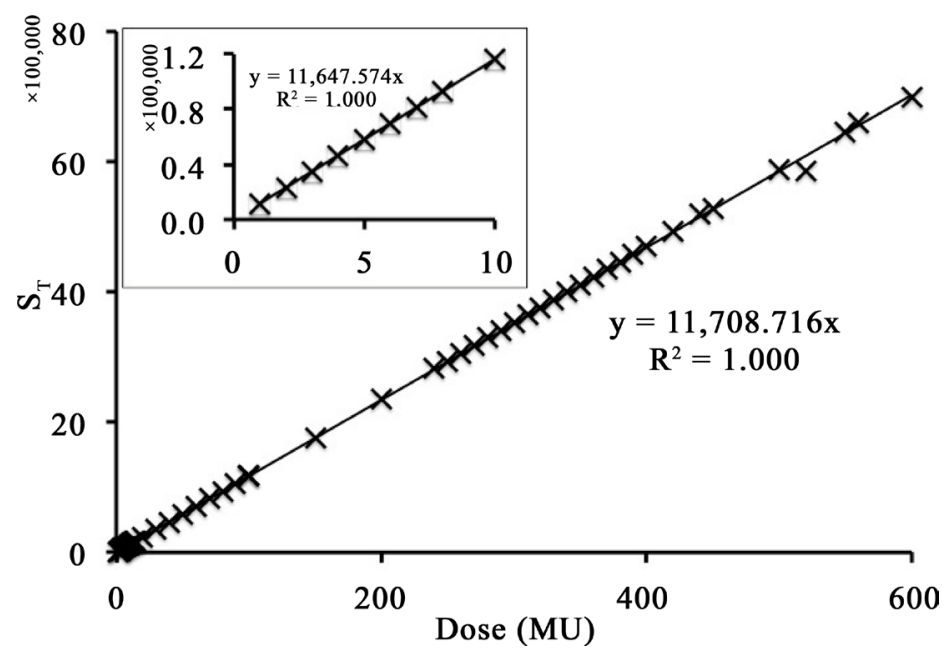

Figure 7. The total integrated signal is linear for the whole range, the small dose range is highlighted in the subset frame. Solid line is the linear fit curve.

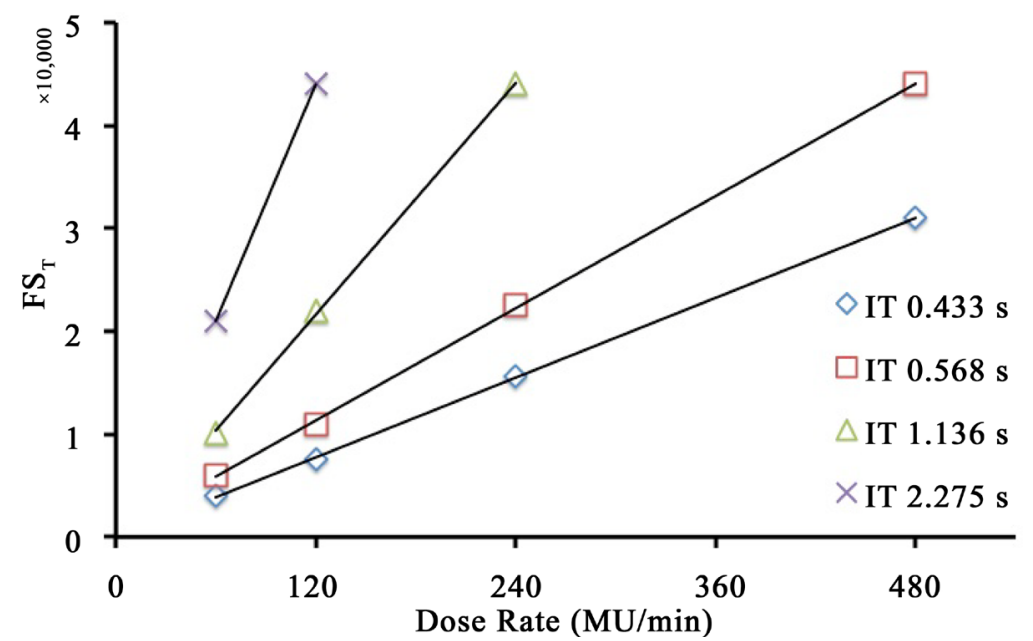

Figure 8. $\mathrm{FS}_{\mathrm{I}}$ as a function of dose rate for the different available integration times. Its value is independent of the radiation dose and depends only on dose rate and integration time. The solid lines represent linear fit curves. 


\subsection{EPID Computer Model}

The computer model results were in agreement with measured data, with the same readout effect observed in EPID measurements, Figure 2 and Figure 9, observable in the computed frame images, Figure 10. These effects, observed in the first and the last few frames, are attributed to the lack of signal contribution from preceding (or subsequent) frames as the radiation has not started (or has ended). Signal profiles from EPID simulation were generally in agreement with the measured ones and demonstrated the same main features. However, there were some differences between the simulation and measured signal profiles, Figure 11. First, it took one or two frames in the simulation to overcome the readout technique effect where the second frame's signal is always very close to $\mathrm{FS}_{\mathrm{I}}$, while it took one or two frames longer in the actual measurement, Figure 11. Second, the residual signal in the simulation results seems to last for a shorter period of time with a lower value than measurement, and the simulated signal profile does not seem to be increasing during irradiation as observed in EPID-1 or decreasing as observed in some EPID-2 measurements. These differences are due to the change in the

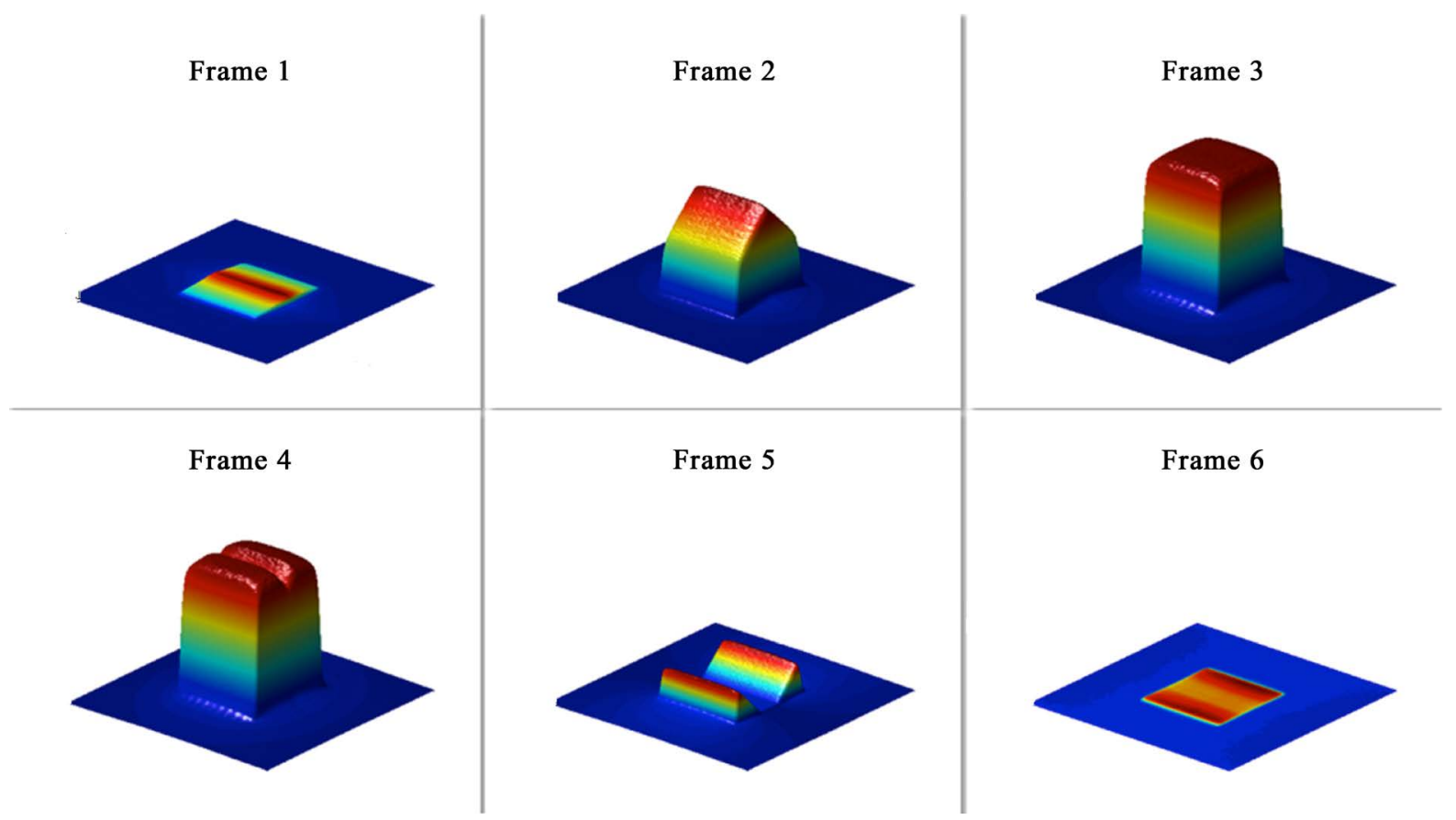

Figure 9. Measured individual two dimensional frame signals appear to start from the centre of the EPID and propagate laterally in the following frames at the beginning of irradiation, and the opposite at the end of irradiation, this is a result of the EPID readout technique.

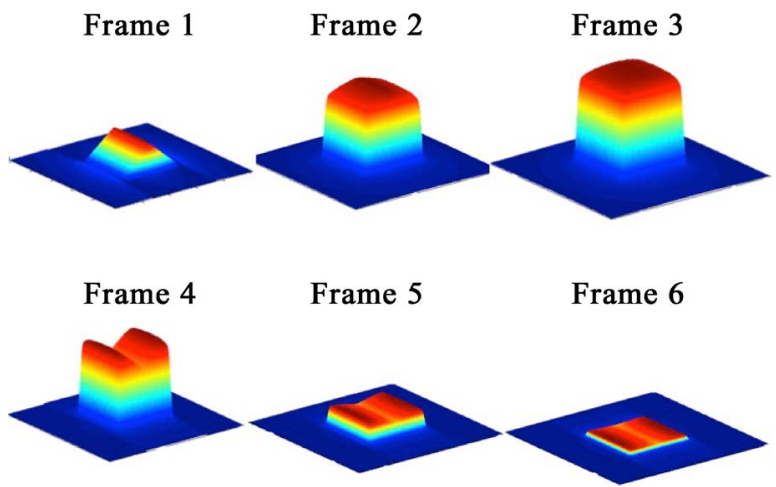

Figure 10. Comuter simulation of the EPID response to radiation designed to study the effect of readout technique on the EPID signal with definable dose, field size, dose rate and integration time. The simulation results were in agreement with experimental results shown in Figure 9. 


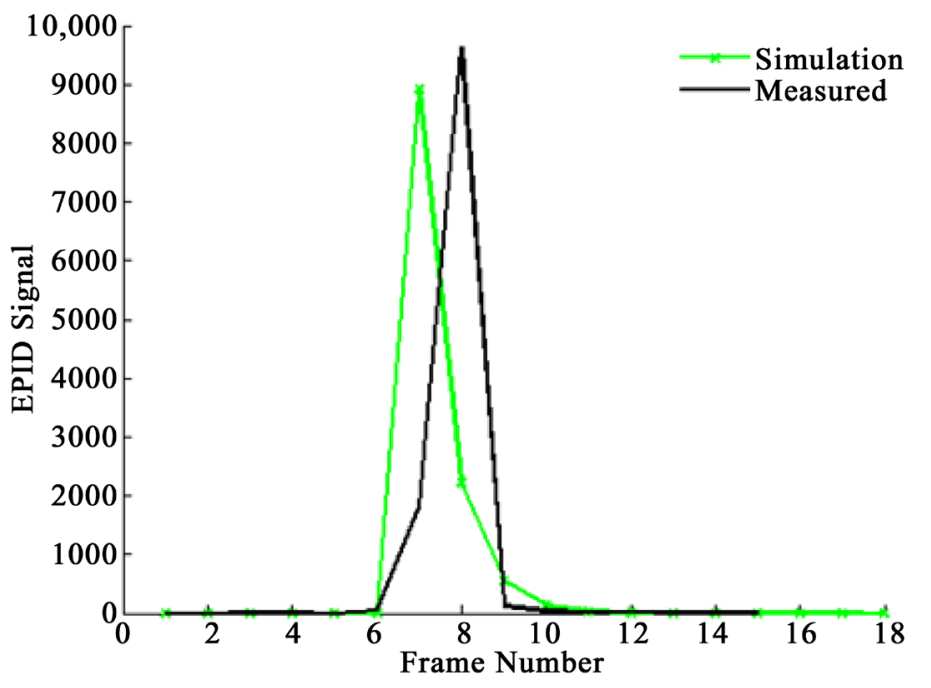

(b)

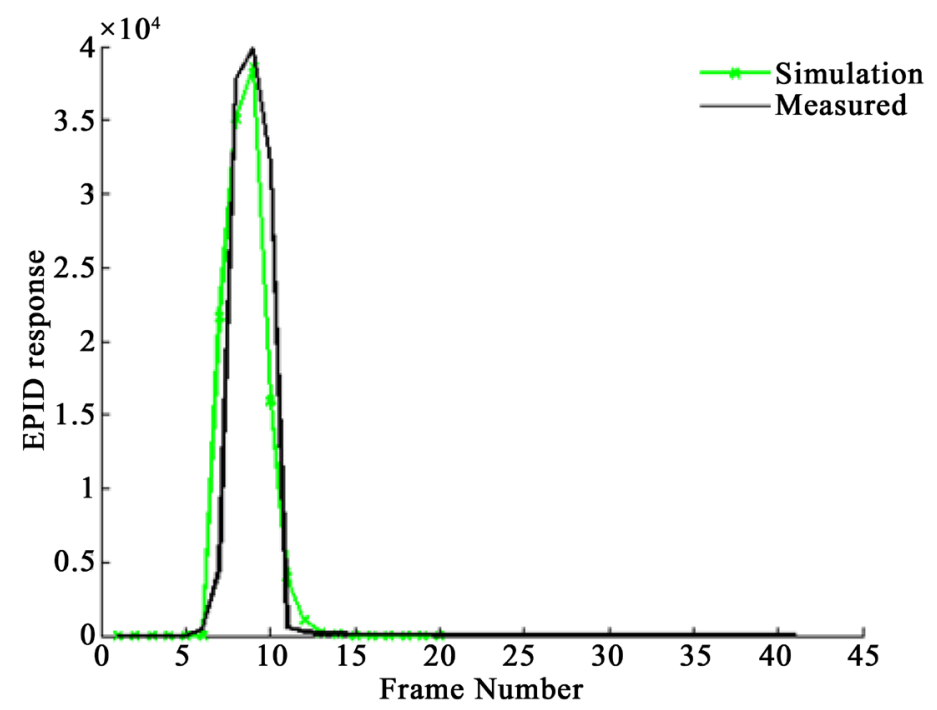

(c)

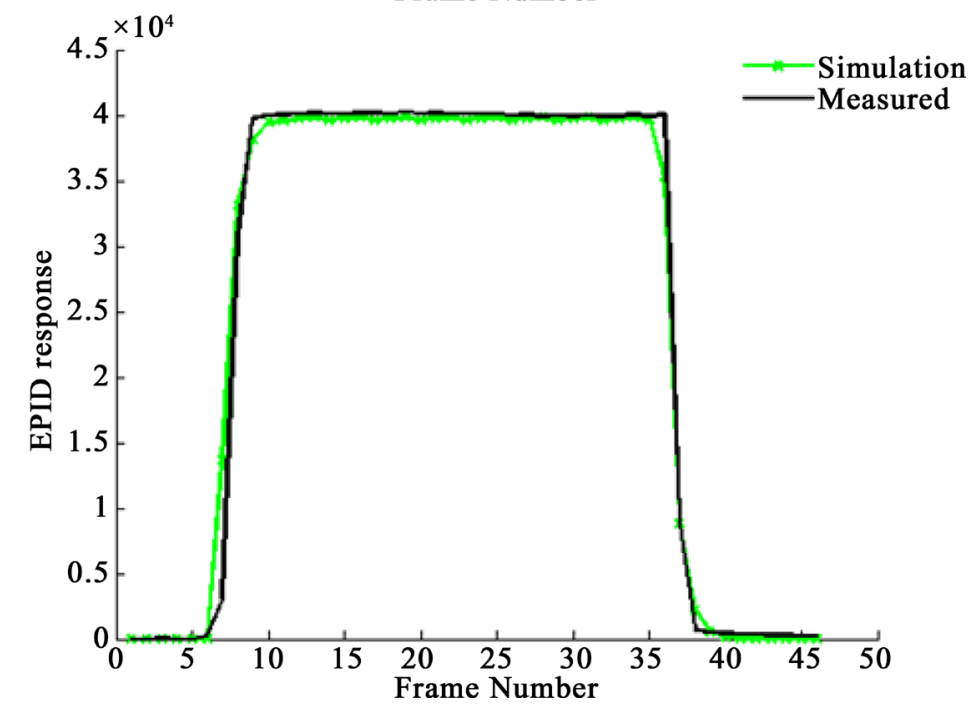

Figure 11. The iViewGT computer model results were in agreement with measurements, the signal profile from simulation in green is compared to that from experimental measurement in black for a radiation dose of $1 \mathrm{MU}$ (a), $10 \mathrm{MU}$ (b) and 100 MU (c) at a dose rate of $480 \mathrm{MU} / \mathrm{min}$ and $0.433 \mathrm{~s}$ integration time. The frame signals are expressed in gray scale values. 
detector's sensitivity as it is being irradiated which was difficult to model accurately. However, the differences between total integrated signal $\mathrm{S}_{\mathrm{T}}$ from simulation and measurement were small $(<1 \%)$ for the whole range of interest. The computer model had demonstrated that the readout technique results in half the EPID measured signal during any given frame being readout in the immediately succeeding frame. At the same time, frame readout signals are a combination of signal measured in the current frame and the immediately preceding one. From these measurements, and computer simulations, it is clear that the shape of the signal profile and the two dimensional frame images are influenced by the readout technique.

\section{Discussion}

\subsection{EPID Signal Profile}

Radiation response characteristics of a-Si based EPID devices have been discussed extensively in the literature. However, the majority of the reviewed literature does not indicate a clear definition for the measured EPID signal either in terms of time duration or number of frames. In addition, the effect of the EPID integration time is often overlooked. One method of quantifying the EPID signal frequently described in the reviewed literature is by multiplying the number of frames by the averaged frame signals. Although the generally reported EPID characteristic in the reviewed literature is the linear EPID dose response [16], some researchers have reported EPID under-response to small radiation doses [3] [4] [6] [8] [11] [12] [14] [15] [17] [20] [21] [24] [26]. Others, however have contradicted that and reported a non-linear or sub-linear EPID dose response [2] [9] [10] [18] [19] [23]. Reported inaccurate EPID dosimetry characteristics may be explained by unclear definitions of the radiation beam properties in terms of EPID parameters.

The terminology implemented in this work to describe and characterize the iViewGT signal profile could provide a workable structure for an a-Si based EPID dosimetry system that includes the calibration process and routine measurements. This EPID dosimetry calibration system is based on a through description of the EPID response signal that accounts for the radiation beam properties and EPID characteristics.

The EPID calibration process aims to correlate $\mathrm{S}_{\mathrm{T}}$ signal magnitude to the Linac MU setting. $\mathrm{S}_{\mathrm{T}}$ must be measured accurately to attain the desired accuracy. However, auxiliary EPID hardware may limit the measurement accuracy of $S_{T}$ with longer irradiations and faster integration times. This limitation can be overcome by using slower integration times in the calibration process to evaluate $\mathrm{S}_{\mathrm{T}}$ since it is independent of the integration time. Another advantage for this EPID dosimetry approach is that the magnitude of measurement uncertainty related to $S_{R}$ measurements could be quantified from the calibration process. The measured data shows that $S_{R}$ comprises less than $2.1 \%$ of $\mathrm{S}_{\mathrm{T}}$ with the highest dose rate and shortest integration time and less than that with lower dose rates, Table 2. Thus, if $S_{M}$ is used to represent the EPID response signal instead of $S_{T}$ the maximum measurement uncertainty would be less than $2.1 \%$. This may be a convenient compromise when such level of accuracy is acceptable. In addition, accuracy of better than $0.5 \%$ is achievable using $\mathrm{S}_{\mathrm{M}}$ with the highest dose rate and shortest integration time for radiation doses more than $200 \mathrm{MU}$, and considerably less for lower dose rates, Table 2. Furthermore, EPID response signal measurement accuracy of approximately $0.2 \%$ could be achieved by including the signals from ten frames after $\mathrm{S}_{\mathrm{M}}$. That means that $\mathrm{S}_{\mathrm{T}}$ is approximated to the magnitude of the sum of $\mathrm{S}_{\mathrm{M}}$ and the ten following frame signals, Table 2. The data presented in Table 2 is obtained from EPID-2 measurements, EPID-1 data for the highest dose rate and shortest integration time had shown similar behavior with slightly higher $\mathrm{S}_{\mathrm{R}}$ value relative to $\mathrm{S}_{\mathrm{T}}$, less than $2.5 \%$.

The EPID signal, from a single pixel or a group of pixels, reflects the radiation beam intensity and geometry.

Table 2. Level of measurement accuracy suitable to the application of interest could be set using the calibration data. If a $2.1 \%$ accuracy level is acceptable, $\mathrm{S}_{\mathrm{M}}$ could be then used instead of $\mathrm{S}_{\mathrm{T}}$ to save time. Higher accuracy levels are achievable when $S_{T}$ is approximated to the sum of $S_{M}$ and the following 10 frames signal.

\begin{tabular}{cccc}
\hline Dose rate $(\mathrm{MU} / \mathrm{min})$ & Maximum $\mathrm{S}_{\mathrm{R}}$ relative to $\mathrm{S}_{\mathrm{T}}(\%)$ & $\begin{array}{c}\text { Threshold Dose for 0.5\% uncertainty in } \\
\mathrm{S}_{\mathrm{T}}(\mathrm{MU})\end{array}$ & $\begin{array}{c}\text { Maximum uncertainty in } \\
\mathrm{S}_{\mathrm{M}}+10 \text { Frames }\end{array}$ \\
\hline 480 & 2.1 & 200 & 0.2 \\
240 & 1.6 & 100 & 0.1 \\
120 & 1.3 & 50 & $<0.1$ \\
60 & 0.8 & 15 & $<0.1$ \\
\hline
\end{tabular}


The Linac MU setting $\left(\mathrm{D}_{\mathrm{MU}}\right)$ used to produce the radiation beam or the radiation dose to the calibration point, i.e. $\mathrm{d}_{\max }$, could be calculated directly from the EPID $\mathrm{S}_{\mathrm{T}}$ signal using Equation (1).

$$
D_{M U}=C \cdot S_{T}
$$

The constant $C$, the reciprocal of $S_{T}$ per $M U$, should be established in the calibration process using a range of measurements sufficient to minimize the statistical uncertainties to an acceptable level.

\subsection{Readout Technique}

The measured readouts from short irradiations that last for a time less than twice the integration time do not generate uniform individual frame images. Such frame signals are a combination of radiation induced charge and baseline signal (zero). For long irradiations, that last for a time longer than twice the integration time, any given frame signal during irradiation will be a combination of radiation induced charge during the current and the previous frames integration times. For a steady dose rate and a given integration time, the value of the signal measured in the previous frame is equal, or very close, to the value of the measured signal in the current frame. This equilibrium between readouts is the reason for the uniform two dimensional frame images during irradiation regardless of the readout cycle, and it explains the irregular field shapes observed in the two dimensional frame images in short irradiations where there is no readout equilibrium.

For a given radiation dose defined by MU setting and dose rate to achieve frame readout equilibrium with a certain integration time, at least one frame readout has to be started after the commencement of irradiation. In addition, the radiation beam has to last at least to the end of the subsequent frame's integration time, because the first frame will always suffer the readout start-up effect and the fact that half the signals in any subsequent frame are generated in the preceding one. In other words, in order to achieve readout equilibrium the irradiation time has to be long enough to accommodate the formation of at least two fames, or more than twice the integration time. The duration of radiation pulses or beam time in seconds can be calculated from the dose and dose rate using Equation (2). The ratio of beam time in Equation (2) to the integration time represents the number of frames that can be formed with that radiation dose, dose rate and integration time combination. This ratio is useful to indicate whether that radiation dose, dose rate and integration time combination will result in readout equilibrium or not. When normalized to the minimum number of frames required for equilibrium it could be used as an equilibrium index that designates frame readout equilibrium with values more than unity, Equation (3).

$$
\begin{gathered}
\text { Beam time }=\frac{60 \times \operatorname{Dose}(\mathrm{MU})}{\operatorname{Dose} \operatorname{Rate}\left(\frac{\mathrm{MU}}{\mathrm{min}}\right)} \\
\text { Equilibrium Index }=\frac{30 \times \operatorname{Dose}(\mathrm{MU})}{\text { Dose Rate }\left(\frac{\mathrm{MU}}{\mathrm{min}}\right) \times \operatorname{Integration} \operatorname{time}(\mathrm{s})}
\end{gathered}
$$

In measurements with equilibrium index less than one, frame readout equilibrium is not achievable. This has been observed in experimental measurements on both EPID-1 and EPID-2, and in the computer model simulation. Although the total integrated signal is uniform, none of the individual frames attains readout equilibrium. On the other hand, measurements with equilibrium index more than one do achieve readout equilibrium. The signal profile in such cases could be divided into three regions based on the readout equilibrium status. The first is the pre-equilibrium phase between the frames $\mathrm{FN}_{0}$ and $\mathrm{FN}_{\mathrm{I}}$. Second, the equilibrium phase starts from the frame $\mathrm{FN}_{\mathrm{I}}$ to the frame $\mathrm{FN}_{\mathrm{G}}$. And lastly, the post-equilibrium phase starting at the frame $\mathrm{FN}_{\mathrm{M}}$ and continuing for several frames.

\subsection{The Residual Signal}

Different definitions and methods to quantify the image lag and ghosting effects on the EPID signal have been proposed. These effects are generally quantified in terms of the measured signal after the end of irradiation [2] [5] [7]-[9] [17] [18] [27]. $\mathrm{S}_{\mathrm{R}}$ is meaningful with equilibrium indices more than unity. In the case where the equilibrium index is less than one, $S_{R}$ cannot be defined because the whole signal profile, or at least part of it, is ob- 
served after the end of irradiation. In such scenarios, few frames are adequate to register all the measurable EPID signals above baseline values.

Although $S_{R}$ is clearly defined on the signal profile, its magnitude is not easy to quantify accurately for two reasons. The first is the hardware capacity that limits $S_{R}$ measurements with long irradiations, and the second is the effect of the readout technique. $\mathrm{FS}_{\mathrm{M}}$ includes the EPID signal from the second half of the previous $\mathrm{FN}_{\mathrm{G}}$ integration time in addition the last remaining Linac pulses delivered during the first half of $\mathrm{FN}_{\mathrm{M}}$ integration time. However, $\mathrm{FS}_{\mathrm{M}}$ includes some delayed residual signal that result from the whole irradiation history and not only that in the last frame [2] [5] [8] [25] [28]. Hence, $\mathrm{FS}_{\mathrm{M}}$ will always contain a part of the residual signal and therefore $S_{R}$ is always under estimated. The amount of delayed residual signal being readout with $\mathrm{FS}_{\mathrm{M}}$ is variable and difficult to estimate since it depends on the coincidence of the completion of frame $\mathrm{FN}_{\mathrm{M}}$ formation and the end of irradiation, similar to the variability of $\mathrm{FS}_{\mathrm{I}}$. However, when considering the rapid decay of the residual signal, shown in the signal profiles in Figure 3, it is perceptible that a considerable amount of the delayed residual signal is potentially included in $\mathrm{FS}_{\mathrm{M}}$. Thus, $\mathrm{S}_{\mathrm{R}}$ is significantly under estimated and its value has a high uncertainty due to the measurement variability associated with the readout technique.

\section{Conclusion}

The noticeable tendency in some of the reviewed published work to conclude inaccurate EPID performance characteristics is mainly a reflection of the arbitrary definition of the radiation beam properties in terms of EPID parameters. That accuracy gets even worse when the EPID functionality and parameters are not fully understood or accounted for. The methodology implemented in this work to describe and characterize the iViewGT signal profile could provide a workable structure for a-Si based EPID dosimetry system that includes the calibration process and routine measurements. This EPID dosimetry calibration system is based on comprehensive description of the EPID response signal that accounts for the radiation beam properties and EPID characteristics.

\section{Acknowledgements}

We would like to express our thanks and gratitude to Dr Abiodun Adeyemi, from the Royal Berkshire Hospital, Reading, UK, and Dr Simon Duane, from the National Physical Laboratory, Teddington, UK, for allowing the experimental work to take place at their facilities, and for the continuous help and support during the course of this work. This work was supported by the Research Centre at King Fahad Medical City, Riyadh, Saudi Arabia (IRB 015-005).

\section{References}

[1] Van Elmpt, W., McDermott, L., Nijsten, S., Wendling, M., Lambin, P. and Mijnheer, B. (2008) A Literature Review of Electronic Portal Imaging for Radiotherapy Dosimetry. Radiotherapy and Oncology, 88, 289-309. http://dx.doi.org/10.1016/j.radonc.2008.07.008

[2] McDermott, L.N., Louwe, R.J.W., Sonke, J.J., van Herk, M.B. and Mijnheer, B.J. (2004) Dose-Response and Ghosting Effects of an Amorphous Silicon Electronic Portal Imaging Device. Medical Physics, 31, 285-295. http://dx.doi.org/10.1118/1.1637969

[3] Nijsten, S.M.J.J.G., van Elmpt, W.J.C., Jacobs, M., Mijnheer, B.J., Dekker, A.L.A.J., Lambin, P., et al. (2007) A Global Calibration Model for a-Si EPIDs Used for Transit Dosimetry. Medical Physics, 34, 3872-3884. http://dx.doi.org/10.1118/1.2776244

[4] McCurdy, B.M.C., Luchka, K. and Pistorius, S. (2001) Dosimetric Investigation and Portal Dose Image Prediction Using an Amorphous Silicon Electronic Portal Imaging Device. Medical Physics, 28, 911-924. http://dx.doi.org/10.1118/1.1374244

[5] Mail, P.O.B. and Pang, G. (2007) Lag Correction Model and Ghosting Analysis for an Indirect-Conversion Flat-Panel Imager. Journal of Applied Clinical Medical Physics, 11, 10.

[6] McCurdy, B.M.C. and Greer, P.B. (2009) Dosimetric Properties of an Amorphous-Silicon EPID Used in Continuous Acquisition Mode for Application to Dynamic and Arc IMRT. Medical Physics, 36, 3028-3039. http://dx.doi.org/10.1118/1.3148822

[7] Sonke, J.-J., Ploeger, L.S., Brand, B., Smitsmans, M.H.P. and van Herk, M. (2004) Leaf Trajectory Verification during Dynamic Intensity Modulated Radiotherapy Using an Amorphous Silicon Flat Panel Imager. Medical Physics, 31, 389-395. http://dx.doi.org/10.1118/1.1639125 
[8] McDermott, L.N., Nijsten, S.M.J.J.G., Sonke, J.J., Partridge, M., van Herk, M. and Mijnheer, B.J. (2006) Comparison of Ghosting Effects for Three Commercial a-Si EPIDs. Medical Physics, 33, 2448-2251. http://dx.doi.org/10.1118/1.2207318

[9] Fidanzio, A., Cilla, S., Greco, F., Gargiulo, L., Azario, L., Sabatino, D., et al. (2011) Generalized EPID Calibration for in Vivo Transit Dosimetry. Physica Medica, 27, 30-38. http://dx.doi.org/10.1016/j.ejmp.2010.02.002

[10] Fidanzio, A., Greco, F., Gargiulo, L., Cilla, S., Sabatino, D., Cappiello, M., et al. (2011) A Generalized Calibration Procedure for in Vivo Transit Dosimetry Using Siemens Electronic Portal Imaging Devices. Medical and Biological Engineering and Computing, 49, 373-383. http://dx.doi.org/10.1007/s11517-010-0699-6

[11] Vial, P., Greer, P.B., Oliver, L. and Baldock, C. (2008) Initial Evaluation of a Commercial EPID Modified to a Novel Direct-Detection Configuration for Radiotherapy Dosimetry. Medical Physics, 35, 4362-4374. http://dx.doi.org/10.1118/1.2975156

[12] Grzadziel, A., Smolinska, B., Rutkowski, R. and Slosarek, K. (2007) EPID Dosimetry-Configuration and Pre-Treatment IMRT Verification. Reports of Practical Oncology \& Radiotherapy, 12, 307-312. http://dx.doi.org/10.1016/S1507-1367(10)60069-7

[13] Slosarek, K., Szlag, M., Bekman, B. and Grzadziel, A. (2010) EPID in Vivo Dosimetry in RapidArc Technique. Reports of Practical Oncology \& Radiotherapy, 15, 8-14. http://dx.doi.org/10.1016/j.rpor.2010.01.003

[14] Awusi, K., et al. (2008) Assessment of Dosimetrical Performance in 11 Varian a-Si500 Electronic Portal Imaging Devices. Physics in Medicine and Biology, 53, 6893. http://dx.doi.org/10.1088/0031-9155/53/23/016

[15] Gustafsson, H, Vial, P., Kuncic, Z., Baldock, C., Greer, P.B. and Denham, J.W. (2011) Direct Dose to Water Dosimetry for Pretreatment IMRT Verification Using a Modified EPID. Medical Physics, 38, 6257-6264. http://dx.doi.org/10.1118/1.3656946

[16] Juste, B., Miró, R., Diez, S., Campayo, J.M. and Verdú, G. (2009) Monte Carlo Simulation of the iView GT Portal Imager Dosimetry. Applied Radiation and Isotopes, 68, 922-925. http://dx.doi.org/10.1016/j.apradiso.2009.10.051

[17] Van Esch, A., Depuydt, T. and Huyskens, D.P. (2004) The Use of an aSi-Based EPID for Routine Absolute Dosimetric Pre-Treatment Verification of Dynamic IMRT Fields. Radiotherapy and Oncology, 71, 223-234. http://dx.doi.org/10.1016/j.radonc.2004.02.018

[18] Winkler, P., Hefner, A. and Georg, D. (2005) Dose-Response Characteristics of an Amorphous Silicon EPID. Medical Physics, 32, 3095-3105. http://dx.doi.org/10.1118/1.2040711

[19] Winkler, P. and Georg, D. (2006) An Intercomparison of 11 Amorphous Silicon EPIDs of the Same Type: Implications for Portal Dosimetry. Physics in Medicine and Biology, 51, 4189. http://dx.doi.org/10.1088/0031-9155/51/17/005

[20] Talamonti, C., Casati, M. and Bucciolini, M. (2006) Pretreatment Verification of IMRT Absolute Dose Distributions Using a Commercial a-Si EPID. Medical Physics, 33, 4367-4378. http://dx.doi.org/10.1118/1.2357834

[21] Wendling, M., Louwe, R.J.W., McDermott, L.N., Sonke, J.-J., van Herk, M. and Mijnheer, B.J. (2006) Accurate Two-Dimensional IMRT Verification Using a Back-Projection EPID Dosimetry Method. Medical Physics, 33, 259273. http://dx.doi.org/10.1118/1.2147744

[22] Wendling, M., McDermott, L.N., Mans, A., Sonke, J.-J., van Herk, M. and Mijnheer, B.J. (2009) A Simple Backprojection Algorithm for 3D in Vivo EPID Dosimetry of IMRT Treatments. Medical Physics, 36, 3310-3321. http://dx.doi.org/10.1118/1.3148482

[23] Liebich, J., Licher, J., Scherf, C., Kara, E., Koch, N., Rödel, C., et al. (2011) Simple Proposal for Dosimetry with an Elekta iViewGT ${ }^{\mathrm{TM}}$ Electronic Portal Imaging Device (EPID) Using Commercial Software Modules. Strahlentherapie und Onkologie, 187, 316-321. http://dx.doi.org/10.1007/s00066-011-2176-Z

[24] Podesta, M., Nijsten, S.M.J.J.G., Snaith, J., Orlandini, M., Lustberg, T., Emans, D., et al. (2012) Measured vs Simulated Portal Images for Low MU Fields on Three Accelerator Types: Possible Consequences for 2D Portal Dosimetry. Medical Physics, 39, 7470-7479. http://dx.doi.org/10.1118/1.4761950

[25] Alshanqity, M., Duane, S. and Nisbet, A. (2012) A Simple Approach for EPID Dosimetric Calibration to Overcome the Effect of Image-Lag and Ghosting. Applied Radiation and Isotopes, 70, 1154-1157. http://dx.doi.org/10.1016/j.apradiso.2012.02.003

[26] Tyner, E., McClean, B., McCavana, P. and Wetterstedt, S. (2009) Experimental Investigation of the Response of an a-Si EPID to an Unflattened Photon Beam from an Elekta Precise Linear Accelerator. Medical Physics, 36, 1318-1329. http://dx.doi.org/10.1118/1.3089424

[27] Partridge, M., Hesse, B.M. and Müller, L. (2002) A Performance Comparison of Direct- and Indirect-Detection Flat-Panel Imagers. Nuclear Instruments and Methods in Physics Research Section A: Accelerators, Spectrometers, Detectors and Associated Equipment, 484, 351-363. http://dx.doi.org/10.1016/S0168-9002(01)02023-X

[28] Zhao, W., De Crescenzo, G. and Rowlands, J.A. (2002) Investigation of Lag and Ghosting in Amorphous Selenium Flat-Panel X-Ray Detectors. SPIE Proceedings, 4682, 9-20. http://dx.doi.org/10.1117/12.465557 